\title{
CASTOR: An Architecture to Bring Cognition to Transport Networks
}

\author{
L. Velasco ${ }^{1 *}$, Ll. Gifre ${ }^{2}$, J.-L. Izquierdo-Zaragoza ${ }^{1}$, G. Julián-Moreno ${ }^{3}$, and J.E. López de Vergara ${ }^{2,3}$ \\ ${ }^{I}$ Optical Communications Group (GCO), Universitat Politècnica de Catalunya (UPC), Barcelona, Spain \\ ${ }^{2}$ Universidad Autónoma de Madrid (UAM), Madrid, Spain ${ }^{3}$ Naudit HPCN, Madrid, Spain \\ e-mail:lvelasco@ac.upc.edu
}

\begin{abstract}
CASTOR architecture to enable cognitive networking is demonstrated. Extended nodes make local decisions, whilst a centralized system beside the network controller makes network-wide decisions. Interaction with ONOS, Net2Plan, and passive monitoring devices is exhibited.

(C) 2018 Optical Society of America

OCIS codes: (060.4250) Networks; (060.4261) Networks, protection and restoration
\end{abstract}

\section{Overview}

Future 5G applications will increase traffic dynamicity and require quality and availability guarantees. These requirements entail large overprovisioning, which increases costs and reduces margin to network operators. The solution to reduce overprovisioning while improving service quality and availability lies in monitoring traffic to be able to predict future conditions that, because known in advance, allow optimization algorithms to find optimal resource allocation, which can be implemented in the network in a proactive manner. In this demonstration, CASTOR, an architecture to enable autonomic networking [1], is shown. CASTOR consists of: i) extended nodes, which collect monitoring data records, pre-process them, and apply data analytics algorithms for local decision-making. Passive monitoring devices will be used to monitor the network at different layers. At the optical layer, a monitoring data generator is used, whereas a real L2/L3 probe from Naudit is used at the packet layer. Naudit's L2/L3 probe is being developed in the framework of the METRO-HAUL EU project to capture $100 \mathrm{~Gb} / \mathrm{s}$ link traffic for network monitoring purposes. For this demonstration, current version of Naudit's probe is able to passively capture traffic at $10 \mathrm{~Gb} / \mathrm{s}$ speed; and ii) a centralized module, which applies network-wide data analytics algorithms, so that decision-making can be implemented with a broader vision. Discovered knowledge can be applied for both proactive and reactive network reconfiguration thanks to the interaction with state-of-the-art software-defined networking (SDN) controllers, e.g., ONOS, and planning tools, e.g., Net2Plan [2]. CASTOR is demonstrated by means of a use case targeting a multilayer MPLS-over-optical network and involving an Observe-Analyze-Act (OAA) loop proactively rerouting optical connections after BER degradation detection, before MPLS connections become affected [3].

\section{CASTOR Architecture}

Fig. 1 overviews the proposed architecture; extended node modules receive IPFIX messages from physical nodes containing data records from observation points. Extended nodes can be deployed as separate elements or run inside those physical nodes with enough computation capabilities. The extended node includes a local knowledge discovery from data (KDD) module that contains KDD applications in charge of handling and processing data records. A KDD manager is the entrance point for KDD applications; it receives IPFIX data records and delivers them to the corresponding KDD application, where they are temporarily stored. Since the granularity of data records received from physical nodes is generally finer than that used to export data toward the centralized system, there is an opportunity to apply data aggregation and analytics techniques directly in the network nodes. Hence, upon the reception of monitoring data records from an observation point, a sample handler in the KDD application aggregates data records of the given type and stores them in a temporal repository. Finally, a KDD process aims to discover patterns inside the data set. Periodically, data records are sent toward the controller.

The network control/management system includes the SDN controller and the centralized data analytics module. Data records and notifications from the extended nodes are received by the CASTOR data analytics module, where a data manager module is in charge of storing them into a scalable multi-master database, e.g., Apache Cassandra, as well as to notify the decision maker module, which in turn executes the corresponding KDD process, optionally, on a large-scale data processing engine like Apache Spark. Additionally, the controller manages monitoring configuration across network nodes and monitoring devices, as well as into KDD applications deployed in the extended nodes, through a dedicated RESTCONF interface. In case that a network reconfiguration is needed, the SDN controller can be also triggered. As such, an illustrative use case of an OAA loop entailing network reconfiguration triggered by state changes of the optical layer will be demonstrated.

\section{Demonstration}

The demonstration proposes a multi-layer MPLS-over-optical network scenario controlled by a single ONOS instance and an instance of the CASTOR cognitive platform. L0 optical nodes are simulated inside ONOS, whereas L2 packet nodes are emulated on the GNS3 simulator to illustrate control and monitoring of real nodes,

The research leading to these results has received funding from the EC through the METRO-HAUL project (G.A. $\mathrm{n}^{\circ}$ 761727), from the Spanish MINECO/FEDER TWINS (TEC2017-90097-R) and TRÁFICA (TEC2015-69417-C2-1-R) projects, and from the Catalan Institution for Research and Advanced Studies (ICREA).

(C) 2018 Optical Society of America. Users may use, reuse, and build upon the article, or use the article for text or data mining, so long as such uses are for non-commercial purposes and appropriate attribution is maintained. 


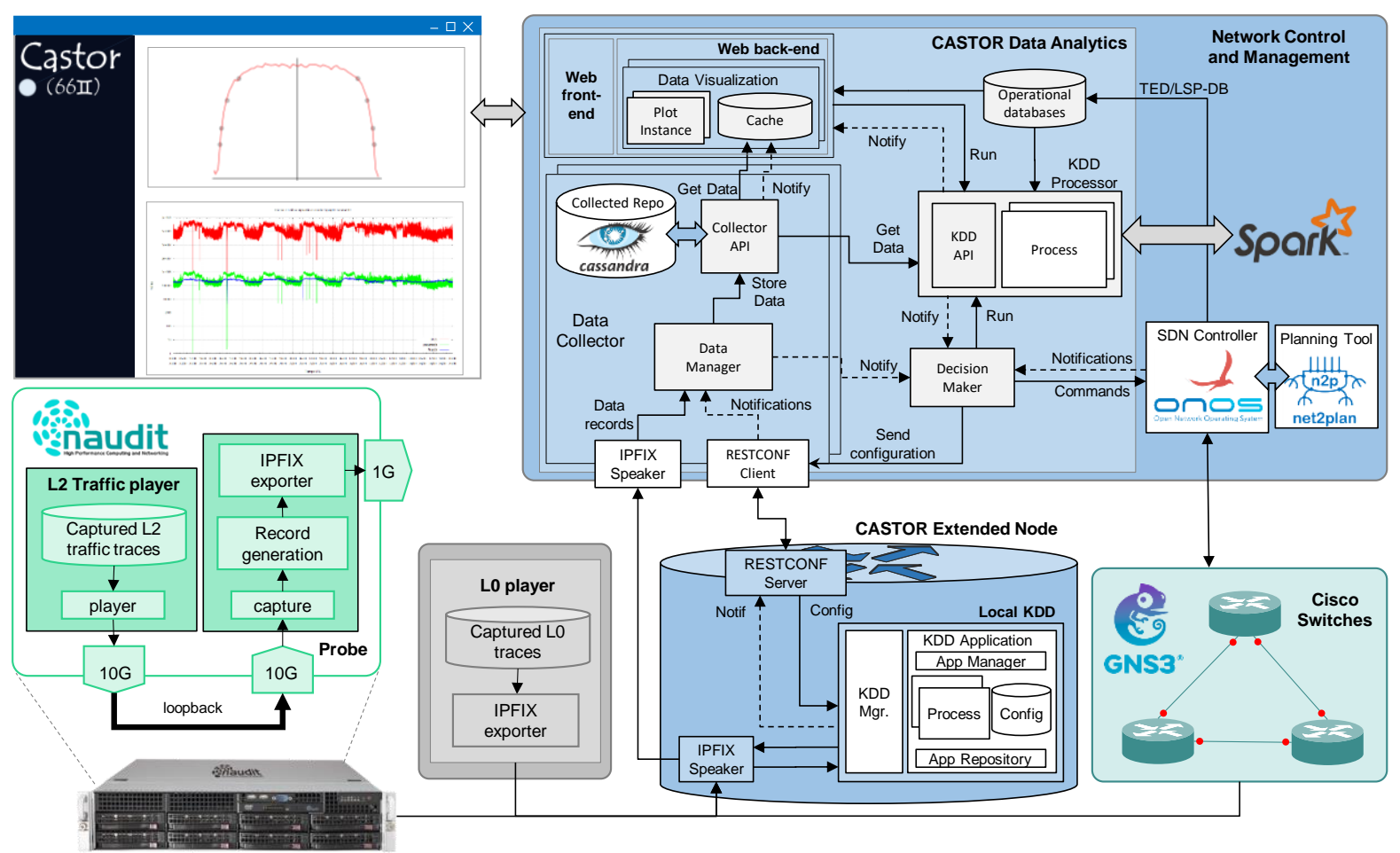

Fig. 1. Demonstration's set-up includes CASTOR's data analytics and extended node.

e.g., based on Cisco IOS. All these devices include monitoring capabilities; specifically, we assume that optical transponders are able to measure BER and received optical power, whilst L2 switches measure packet traffic, e.g., received and transmitted bytes and Ethernet frames. Besides, optical spectrum analyzers (OSA), installed in optical links, are able to monitor the spectrum of the optical connections, whereas L2/L3 probes are able to measure aggregated traffic. Naudit's L2/L3 probe captures and processes packets generating one data record per second with bytes and frames information. Passive probes can be attached to a core switch, where a mirror port or switched port analyzer (SPAN) is enabled to capture all traversing traffic. Given that such core switch is not available for a live demonstration, a traffic player will be used; stored traffic traces will be generated at $10 \mathrm{~Gb} / \mathrm{s}$ data rate and captured in the probe. Such traffic player runs in the same equipment, so a loopback link is established, connecting traffic player and probe interfaces. Finally, those available records are exported using IPFIX to the CASTOR extended node to be further processed.

The workflow of the demonstration will be as follows: $i$ ) CASTOR imports operational databases, i.e., TED and LSP-DB, from ONOS; $i$ ) an operator activates monitoring of an optical connection (transponder and OSA), an MPLS LSP, and a L2 virtual link (L2 Naudit's probe). Measurements are received in CASTOR's extended nodes and collated in the data analytics module; iii) Monitored data will be visualized in the CASTOR platform; $i v$ ) optical connections' BER is continuously measured and locally analyzed in the extended node; unexpected slight BER variations are detected and notifications are sent to the CASTOR data analytics node, which correlates with others received from different connections in the network to discover common failure causes; $v$ ) upon detecting a common link causing increased BER on several optical connections, CASTOR data analytics decides to trigger rerouting of the affected lightpaths through ONOS, before degradation becomes severe. ONOS will be assisted by Net2Plan planning tool with computation of (near) optimal lightpath rerouting.

\section{Innovation and Relevance to OFC Conference}

CASTOR aims at bringing cognitive capabilities to state-of-the-art SDN controllers to create a real autonomic orchestration platform. In the demonstration, integration with passive monitoring devices and with ONOS will be shown. To this end, custom extensions have been developed for both frameworks extending their base services and interfaces, namely: $i$ ) ONOS north interface to export TED and LSP-DB; and ii) decision makerONOS east-west interface to trigger network reconfiguration from the decision maker. Additionally, ONOS is connected to an instance of Net2Plan for path computation and network re-optimization, through a new eastwest interface. The demonstration exhibits timely innovations adding monitoring and data analytics capabilities to existing SDN controllers, for cognitive control and management of complex network scenarios. Hence, the demonstration will attract the community's attention and it will be of interest to a broad OFC audience.

\section{References}

[1] L. Velasco et al., "An Architecture to Support Autonomic Slice Networking," IEEE/OSA JLT, 2018.

[2] P. Pavon-Marino et al., "Net2Plan: An Open Source Network Planning Tool for Bridging the Gap between Academia and Industry," IEEE Network, 2015.

[3] A. P. Vela et al., "BER Degradation Detection and Failure Identification in Elastic Optical Networks," IEEE JLT, 2017. 